\title{
Robotic Laparoendoscopic Single Site Surgery (R-Less) For Bilateral Tubal Obstruction: A Case Report
}

\author{
Hsiang Chun Dong, Mu Hsien Yu and Cheng Chang Chang* \\ Department of Obstetrics and Gynecology, Taiwan
}

Received: 㯺January 09, 2019; Published: 監January 18, 2019

*Corresponding author: Cheng Chang Chang, Department of Obstetrics and Gynecology, Tri Service General Hospital, National Defense Medical Center, Taiwan

\begin{abstract}
Objective: Robotic technology is being increasingly adopted in gynaecologic surgeries. Features such as tremor filtration, motion downscaling, three-dimensional visualization, and multiple degrees of freedom are able to overcome the technical difficulties inherent to laparoscopic tubal anastomosis. We present a case of tubal anastomosis and fimbrioplasty using robotic laparoendoscopic single site surgery (R-LESS).

Case Report: A 31-year-old woman presented with hydrosalpinx of the left fallopian tube and total obstruction of the right fallopian tube. The patient underwent left fimbrioplasty and right tubal anastomosis with R-LESS. The patient tolerated the procedure well with minimal blood loss, and no complications occurred.

Conclusion: Hysterosalpingography was performed 23 days postoperatively, which revealed normal contrast medium filling with peritoneal spill of the right fallopian tube and patency of the left fallopian tube. R-LESS seems to be an applicable alternative technique for tubal anastomosis and fimbrioplasty in selected patients with tubal obstruction.
\end{abstract}

Keywords: Robotic Surgery; Single Site; Tubal Anastomosis; Tubal Fimbrioplasty; Tubal Obstruction

\section{Introduction}

Hydrosalpinges are a common cause of female infertility and are primarily induced by chlamydia and gonorrhoea infections [1]. Women diagnosed with hydrosalpinges can undergo tubal anastomosis or in vitro fertilization (IVF) [2]. However, IVF is expensive, time-consuming, and carries the risk of multiple gestations and drug side effects, and anastomosis may be a better choice. Laparoendoscopic single-site surgery (LESS), an innovative, speedily evolving, minimally invasive technique that requires only an opening at the umbilicus, has become increasingly prevalent [3]. Robotic LESS (R-LESS) has several additional advantages, including shorter surgical time, fewer technical difficulties due to enhanced ergonomics, and the minimization of or decrease in surgeon fatigue [4]. Here, we present a case of bilateral tubal obstruction that underwent R-LESS.

\section{Case Report}

A 31-year-old woman without any prior systemic disease, including pelvic inflammatory disease, presented with an inability to conceive. Hysterosalpingography showed hydrosalpinx of the left fallopian tube and total obstruction of the right fallopian tube (Figure 1A). Due to her religious beliefs, the patient and her husband refused to undergo IVF, and the patient provided written informed consent to publish and elected to undergo surgery.

\section{Surgical Technique}

The da Vinci single site surgery system (Intuitive Surgical, Sunnyvale, CA, USA) was used for this procedure. The robotic tower was positioned between the patient's feet. The abdominal wall and vagina were sterilized, and the patient was placed under general anaesthesia in the lithotomy position with the arms tucked to each side. A 2-cm-long incision was made longitudinally at the umbilicus to access the surgical site. The robotic instruments were introduced into the patient's body to assess the pelvic status and feasibility before beginning surgery. Adhesiolysis was performed to free the left fallopian tube, after which methylene blue dye was injected, filling the hydrosalpinx. A cruciate incision was made on the left hydrosalpinx, and PDS 7/0 sutures were used to reconstruct the edges of the left fallopian tube. 
We observed a paratubal cyst approximately $5 \mathrm{~cm}$ in diameter and a Morgagni cyst on the right adnexa, which were removed. After excision of the right fallopian tube, the ureteral catheter was inserted through the fimbria into the distal fallopian tube. The obstruction at the proximal third of the right fallopian tube was removed, and end-to-end anastomosis was performed with a PDS 7/0 suture. Fimbrioplasty was performed with four PDS 7/0 sutures to reconstruct the right fallopian tube. At the completion of the procedure, the entire pelvis and operation site were irrigated thoroughly to ensure proper haemostasis. Blood loss was minimal, and the patient tolerated the procedure well. The patient had an uneventful recovery and was discharged 48 hours after surgery. Hysterosalpingography performed 23 days postoperatively revealed normal contrast medium filling with peritoneal spill in the right fallopian tube and patency of the left fallopian tube (Figure 1B).

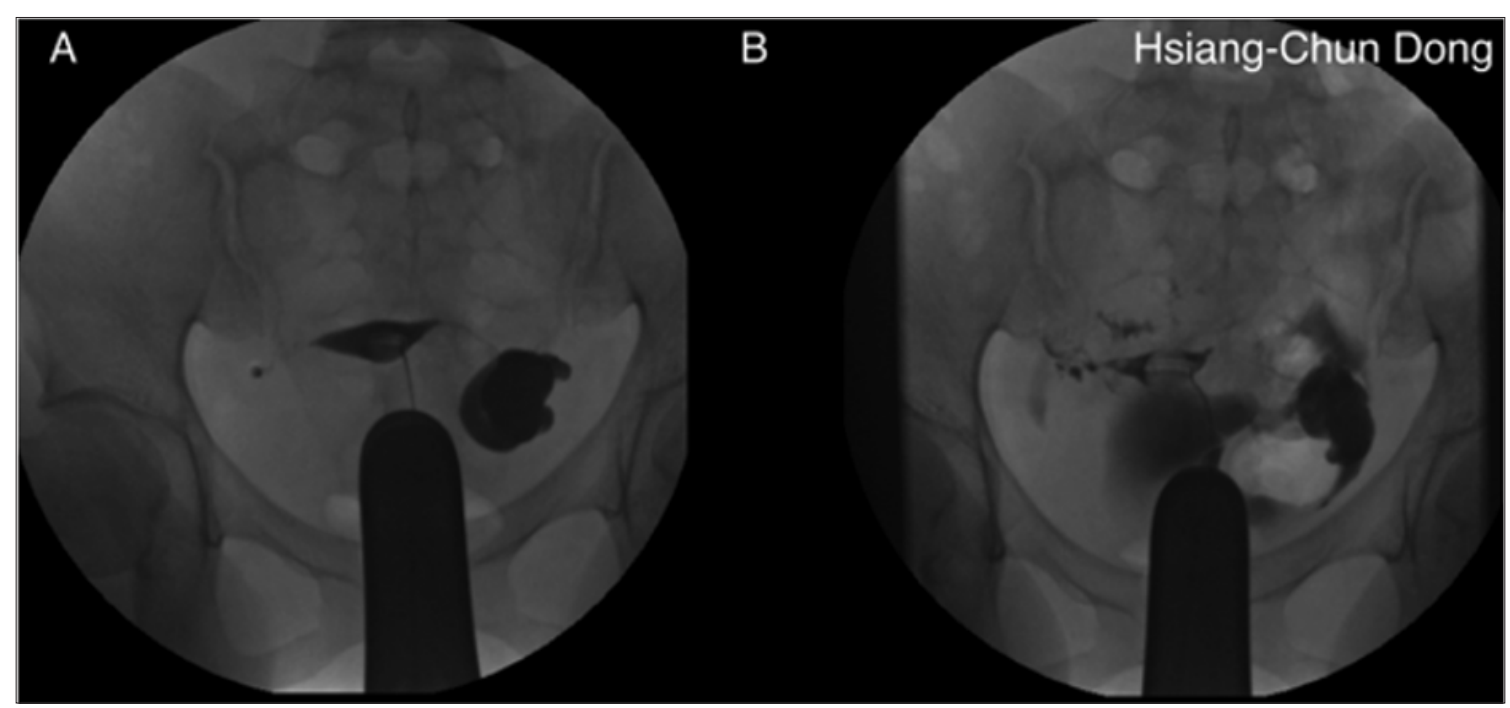

A. Revealed hydrosalpinx of the left fallopian tube and total obstruction of the right fallopian tube.

B. Postoperative hysterosalpingography revealed normal contrast medium filling with peritoneal spill in the right fallopian tube and patency in the left fallopian tube.

Figure 1: Hysterosalpingography.

\section{Discussion}

The laparoscopic approach produces a better reproductive outcome than microsurgery by laparotomy, and has significant advantages, such as less postoperative discomfort, fewer complications, a smaller incisional wound, a shorter recovery time, and earlier resumption of normal activities [5]. Tremor is a significant factor in laparoscopic tubal microsurgery. The distance of the pelvic organs from the trocar sites amplifies tremors and can make micro-suturing difficult. Furthermore, in laparoscopic procedures, the surgeon must adopt an anti-ergonomic position that leads to rapid fatigue [6]. The robotic system facilitated the successful completion of this case by reducing fatigue, filtering tremors, scaling movements, and enabling a constant view of the surgical site.

The da Vinci instrument allows for three-dimensional rotation, providing greater flexibility than a human wrist, allowing for complete reproduction of the surgeon's movement. The sitting position is comfortable, and the chair's arm provides stability during the procedure. Patients may also prefer this approach, as the healed incision remains hidden at the base of the navel. The success of robotic surgery depends on teamwork. The proper establishment of a robotic gynaecologic surgery team is an important concern [7]. The robotic system does not replace surgical skill, but enhances it.
Establishing and integrating the training for robotic surgery into the training program of general gynaecology is advised. R-LESS appears to be feasible and safe for performing tubal anastomosis and fimbrioplasty.

\section{References}

1. Pereira N, Pryor KP, Voskuilen Gonzalez A, Lekovich JP, Elias RT, et al. (2017) Ovarian response and in vitro fertilization outcomes after salpingectomy: does salpingectomy indication matter? J Minim Invasive Gynecol 24(3): 446-454.

2. Kim JH, Jeong CJ (2014) Single-Port Laparoscopic Tubal Anastomosis. CRSLS.

3. Fader AN, Levinson KL, Gunderson CC, Winder AD, Escobar PF (2011) Laparoendoscopic single-site surgery in gynecology: A new frontier in minimally invasive surgery. J Minim Access Surg 7(1): 71-77.

4. Kavoussi SK, Kavoussi KM, Lebovic DI (2014) Robotic-assisted tubal anastomosis with one-stitch technique. J Robot Surg 8(2): 133-136.

5. Falcone T, Goldberg J, Garcia Ruiz A, Margossian H, Stevens L (1999) Full Robotic assistance for laparoscopic tubal anastomosis: a case report. J Laparoendosc Adv Surg Tech A 9(1): 107-113.

6. Göçmen A, Sanlikan F, Uçar MG (2013) Robot-assisted tubal reanastomosis: Initial experience in a single institution. TJOG 52(1): 7780.

7. Shun JT, Chi KL, Pei TF, Yung LL, Cheng CS, et al (2012) Robotic surgery in complicated gynecologic diseases: Experience of Tri-Service General Hospital in Taiwan. TJOG 51(1): 18-25. 


\section{ISSN: 2574-1241}

DOI: $10.26717 / B J S T R .2019 .13 .002388$

Cheng Chang Chang. Biomed J Sci \& Tech Res

(C) (P) This work is licensed under Creative

Submission Link: https://biomedres.us/submit-manuscript.php

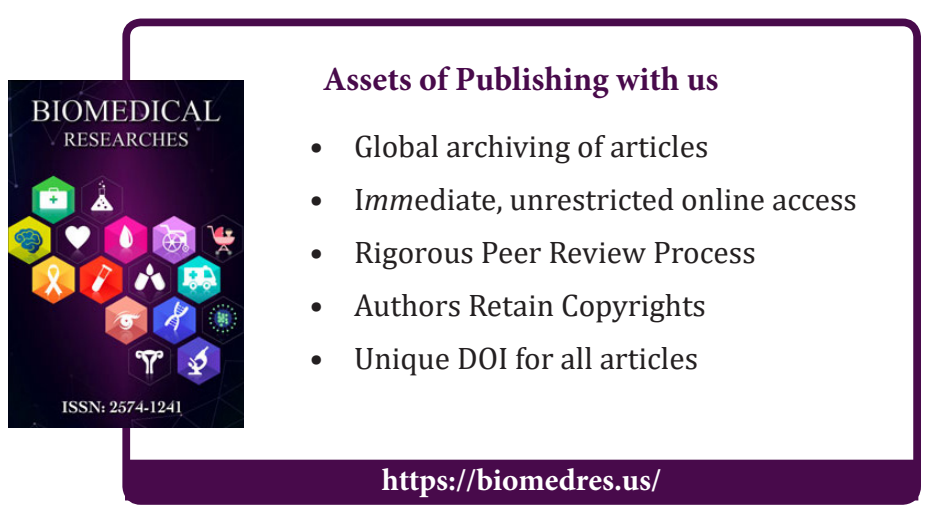

